use, which impart increased precision to the results. Michel-Lévy's elaborate discussion of the optical properties of the felspars has greatly facilitated the discrimination of the several varieties; while among special instruments we may recall Fedorow's "universal theodolite," enabling accurate optical measurements to be made on crystals in random sections. The application of dense liquids to determine the specific gravity of minute fragments of minerals, and to obtain pure material for analysis, has been perfected, and, especially in the form of Sollas's "diffusion column," has been made both simple and convenient for use. The importance of accuracy and completeness in the chemical analysis of rocks has become more generally recognised. Many of the most valuable rock-analyses published in late years are due to the United States Geological Survey, who have realised the importance of maintaining an adequate staff of skilled chemists. One point brought out is the wide distribution in igneous rocks of small amounts of the heavy metals, and of some other elements, such as barium and strontium. Apart from its obvious application to questions concerning the origin of metalliferous deposits, information of this kind will probably be found to throw some light upon matters of more strictly petrological interest.

\section{THE DISAPPEARANCE OF IMAGES ON PHOTOGRAPHIC PLATES.}

$\mathrm{T}$ is the aim of the modern astronomer to employ photography, whenever possib!e, in the many branches of his work, as by this means the peculiarities of the observer are eliminated and a permanent record is obtained that can be examıned at leisure at any later date. In some kinds of work photography helps us in obtaining a great number of facts in a very short space of time, facts which would have taken weeks to accumulate by the old method of eye observation. Not only is the science more rapidly advanced by the greater abundance of material at hand, and therefore available for discussion, but the application of photography to astronomy has opened up so many new fields of work that the whole subject has now a far wider horizon than before.

To be able to photograph in a few hours objects which for ever will be outside the reach of the human eye, however aided, is one of the many marvels of this invaluable method.

The fine photographs obtained to-day, and so well illustrated in our books by the aid of the modern processes of reproduction, suggest the importance of recording the appearances of these celestial objects after some years have elapsed, in order to be able to note whether any changes in brightness or form are occurring. The magnificent work of the "Carte du Ciel," started by the far-seeing mind of Admiral Mouchez, is one of those schemes on a large scale for obtaining a survey of the universe at the close of the nineteenth century. The many thousands and thousands of photographic plates that will have been exposed to the sky when this plan will have been accomplished point out to us the immense importance that must be attached, not only to the "keeping" qualities of the film on the glass plates, but also to the retention of the images on these films.

Since one of the chief objects of Admiral Mouchez's plan was to hand down to posterity a chart of the celestial vault as recorded in our time, to enable those that follow us to compare with it that recorded in their time, the importance of the preservation of the photographic films, and the images impressed on them, cannot be overrated.

Most of the readers of NATURE are familiar with those beautiful photographs of long exposure of nebulæ and star clusters which we owe to the skill and patience of Dr. Isaac Rolserts, and which he has given to the world in two magnificent volumes of plates. In the second of these volumes Dr. Roberts makes some very interesting and valuable remarks regarding the "fading" of some of the images on the photographic plates, and his experience shows that such disappearances of images after long intervals of time may assume very considerable proportions.

Although the period over which his experience extended only covered about ten years, this interval of time was sufficiently long to enable him to obtain some very striking facts.

The following brief summary of the two instances which he recorded in his second volume will give the reader a general quantitative idea of the disappearances of images during a short period.

A photograph of a region of the sky was taken on February 15 in 1886 , and 403 star-images were counted on the negative. A re-count on May 29,1895 , found only 272 images, a loss of 131 images in about nine and threequarter years.

Again, another photograph of identically the same region, taken on Wlarch 22,1886 , recorded 364 stars; the same plate, examined in May 1895 , showed only 234 .

These facts, then, indicate that, even after so short a period as ten years or so, the photographic film cannot be depended on, and that for lasting purposes recourse must be made to some means of reproducing the photographs soon after they have been obtained, the reproductions being as faithful to the originals as possible. It is generally known that, in nearly every method of reproduction, very faint details are lost in the process, but this loss could be easily recorded by noting the slight differences at the time; printed on good paper and with permanent ink, such reproductions should be lasting.

The fact that the great photographic chart of the sky is approaching completion causes onc to think of the great expense that will be involved in reproducing the large number of individual plates. That many of the observatories which have taken part in this undertaking will find some difficulty in at once getting together the necessary funds is quite possible, so that a delay of a few years may be detrimental to the accuracy of the chart.

It is therefore very important that some means should be at hand to prevent the images of the fainter objects from fading quite away, or, if already disappcared, to bring them back to view.

Fortunately, the latter alternative can be accomplished by a process of manipulation which we owe to Sir William Crookes.

The complcteness and success of this method will be gathered from the fact that when Sir William Crookes had "revived" one of Dr. Roberts' negatives that had entirely lost many images, the latter, on re-counting, "found that every one of the missing images had been restored to view, as distinctly, I think, as they were after the negatives were first develnped."

The process by which Sir William Crookes accomplished this has been published in detail by Dr. Roberts in the Monthly Notices of the Royal Astronomical Society (vol. lxi., No. I) for last November. As the description should prove of interest and service to many of our readers, the following account is appended, as described by Sir William Crookes himself in a letter to Dr. Roberts.

"(I) Soak the plate for three hours in distilled water.

(2) Prepare, in advance, two solutions A and B. Solution A.

Pyrogallic acid......... I oz. Sodium metabisulphite I oz.

Water ................... $80 \mathrm{oz}$
Sodi u m carbonate (crystals) .............. 12 oz. Sodium sulphite........ $4 \mathrm{oz}$. Water ................. $80 \mathrm{oz}$ NO. I629, vOL. 63] 
"Mix equal parts of $\mathrm{A}$ and $\mathrm{B}$, and allow the plate to soak in the mixture for ten minutes or a quarter of an hour, in the dark. Wash well.

"(3) Transfer the washed plate to a solution of three oz. of sodium hyposulphite in $20 \mathrm{o} z$. of water. Allow it to remain for half an hour, and then wash the plate in running water for three hours.

"(4) Prepare a 'clearing' solution according to the following formula :-

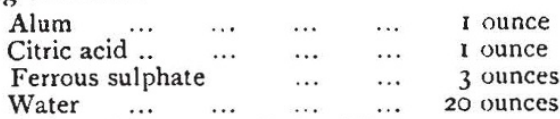

"Allow the plate to soak in this for ten minutes, and then remove and wash in running water for six hours.
"The sulphocyanıde and gold solution has the property of precipitating gold on the image, and rendering it of a blacker colour and diminishing the chance of fading. I should think you will find it useful always to use the clearing solution and the sulphocyanide and gold solution in your usual process." WILLIA.M J. S. LOCKYER.

\section{VIBRATION OF GUN-BARRELS.}

THIS research on the vibration of gun-barrels is a continuation of former investigations on the nature of vibrations set up in a gun-barrel when fixed, with a view to discover how the error of departure is affected by rapid oscillations of the barrels when firmly clamped. The authors' experiments were made on three small-bore

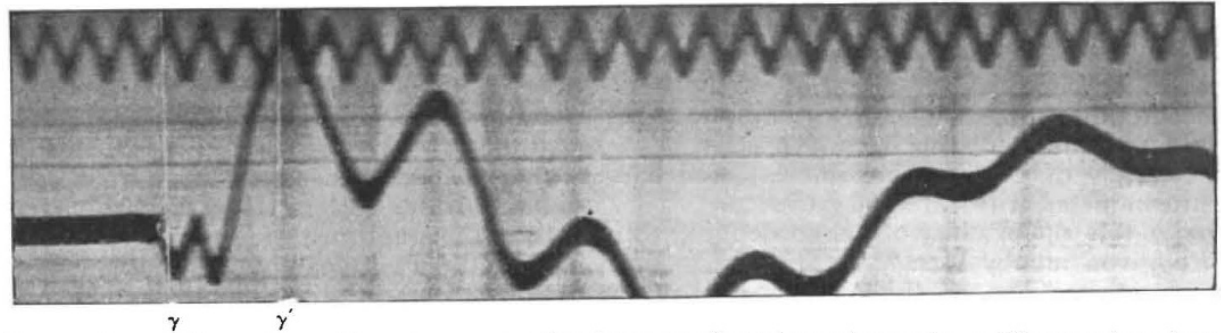

FIG. r. $-3 \mathrm{~mm}$. Cal. Run clamped in cork support. Vibration curve of a point at the muzale. $\gamma$ Disengaged ipark at the instant at which the shot leaves the muzzle; $\gamma^{\prime}$ Iisengaged spark at $4{ }^{\circ} \mathrm{s}$. distance from the muzple. In the unconstrained method of holding the weapon, the amplitude of the vibration is not so strongly marked as when it is clamped. (See Fig. 2.)

“(5) Prepare in advance two solutions, C and D. So'ution C. Ammonium sulphoSoluti in I).
(iold chloride ........... $15 \mathrm{gr}$. Water..... $100 \mathrm{gr}$. Water ................... $15 \mathrm{oz}$

"For use take $I$ ounce of each, and add $\delta$ ounces of water. Soak the plate in this mixture for ten minutes, and at the end of the time remove and wash it in running water for half an hour. Transfer to a dish of distilled water, where it may remain for an hour. Finally, drain on blotting-paper, and allow to dry.

"The separite solutions, A, B, C and D, will keep for an indefinite tim?, and the same may be said of the rifles, placed at their disposal by the firm of Mauserviz., one $8 \mathrm{~mm}$. experimental rifle furnished with a wood stock, one $7 \mathrm{~mm}$. Spanish model, and one trial gun $6 \mathrm{~mm}$. -in all cases smokeless powder was used. In their early experiments the rifles were clamped firmly when fired, but in their recent work this method of support was abandoned, as they found that the clamping imposed restraints which altered the character of the shooting of the rifles and also their mode of vibration. 'This is by no means a new discovery. The late W. E. Metford, of well-known rifle repute, showed long ago $(1870)$ that the shooting of a rifle was greatly changed when the barrel was securely clamped to a heavy mass of iron. In order

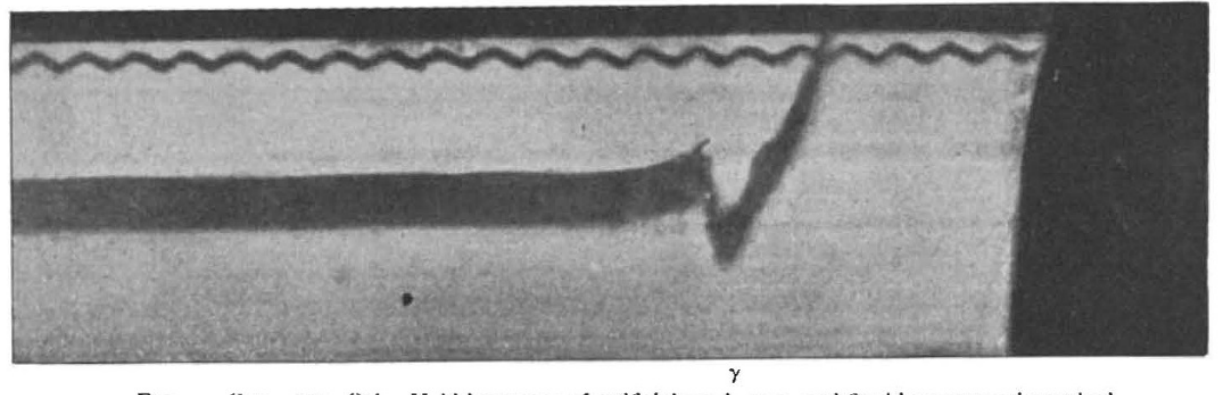

Fig. 2.-Gun $7 \mathrm{~mm}$. Cal. He:d hy means of artificial marksman, and fired by pneumatic method.

clearing solution, if kept tightly corked. But when mixed together they will not keep, so fresh mixtures should be made each time.

"I have given you the full process adopted on the plates you sent me, but I think some of them may be omitted with no disadvantage. For example, I should like to try if the soaking in hyposulphite may be dispensed with. I think it can, but I only tried leaving it out on the plates you sent that had not faded.

"I always found the great secret of preventing images from fading out was to wash them very well in running water. The clearing solution allows the time of washing to be a little shortened, but not much.

No. 1629, voL. 63$]$ to avoid this source of trouble the experimental guns were sometimes embedded in cork and sometimes suspended pendulum fashion by ropes, so that they were practically unrestrained and the condition of support approximated to the normal one, namely, the rather free support of the hands and shoulder of the marksman. The movements of the barrels were recorded by means of photography on a moving sensitive plate. Two methods were employed for firing the guns, viz., an electro-magnetic trigger-puller, and a pneumatic method in which a small piston, moving in a cylinder attached to 1 "Untersuchung über die Vibration des Gewehrlaufes." Von C. Cranz unld K. R. Koch. Pp. $23 ; 4$ plates. (München, 1900.) 